\title{
First-principles prediction of enhanced magnetic anisotropy in FeCo alloys
}

\author{
Dangxin Wu, ${ }^{1}$ Qiming Zhang, ${ }^{1, a)}$ J. Ping Liu, ${ }^{1}$ Dingwang Yuan, ${ }^{2}$ and Ruqian $\mathrm{Wu}^{2}$ \\ ${ }^{1}$ Department of Physics, University of Texas at Arlington, Arlington, Texas 76019-0059, USA \\ ${ }^{2}$ Department of Physics and Astronomy, University of California, Irvine, California 92697-4575, USA
}

(Received 25 October 2007; accepted 12 January 2008; published online 4 February 2008)

\begin{abstract}
The structural, electronic, and magnetic properties of $\mathrm{FeCo}$ alloys were studied by first-principles calculations. It has been found that the alloys prefer chemically noncubic geometries in a wide composition range. This produces appreciable uniaxial magnetic anisotropy, which facilitates interphase magnetic interaction and enhances the overall magnetization in exchange-coupled nanocomposite systems. Large magnetostrictive coefficients provide another venue for manipulations of magnetic anisotropy energies. (C) 2008 American Institute of Physics.
\end{abstract}

[DOI: $10.1063 / 1.2840721]$

Exchange-coupled systems with soft and hard magnets based on the exchange-spring mechanism have been extensively studied to attain high maximum energy product values, $(\mathrm{BH})_{m}{ }^{1}$ As demonstrated in magnetic composites and magnetic thin films, $(\mathrm{BH})_{m}$ can be significantly enhanced by combining high saturation magnetization of soft magnetic phases and high anisotropy of hard magnetic phases. However, the integration of soft magnetic materials is often limited by the thickness of the soft phase for effective exchange coupling between the hard and soft phases. It has been shown that the replacement of a soft phase $\left(K_{1}=0\right)$ by a suitably aligned semihard phase $\left(0<K_{1}<K_{h}\right)$ is beneficial in permanent-magnet nanostructuring ${ }^{2-4}$ but the challenge is to find semihard materials that combine moderate anisotropy with a high magnetization. Traditional $\mathrm{Fe}_{1-x} \mathrm{Co}_{x}$ alloys are ideal candidates for hard-soft nanostructuring ${ }^{5,6}$ because they have a very high magnetization in a wide range of Fe-rich compositions. However, their anisotropy is typically very low $\left(K_{s} \sim 0\right)$ or its development requires a huge and difficultto-realize tetragonal strain $c / a \sim 1.2 .^{7,8}$ For the rational design of optimal exchange-coupled magnetic materials, it is essential to study the magnetic anisotropy and magnetostriction of $\mathrm{Fe}_{1-x} \mathrm{Co}_{x}$ alloys in a broad composition range.

In this paper, we report results of systematic density functional studies of structural and magnetic properties of $\mathrm{Fe}_{1-x} \mathrm{Co}_{x}$ alloys in the Fe-rich stoichiometry. It is interesting that they adopt noncubic structures in $0.18<x<0.31$. The magnetostrictive coefficients $\lambda_{001}$ and $\lambda_{111}$ are sizeable for the stoichiometric $B 2 \mathrm{FeCo}$ alloy. As a result, $\mathrm{Fe}_{1-x} \mathrm{Co}_{x}$ alloys possess appreciable magnetic anisotropy energies.

We used both the Vienna ab initio simulation package (VASP) (Ref. 9) and the full potential linearized augmented plane wave (FLAPW) methods ${ }^{10}$ to solve the density functional Kohn-Sham equations. The spin-polarized generalized gradient approximation was adopted for the description of exchange-correlation interactions among electrons. ${ }^{11}$ In the VASP calculations, the projector augmented wave method $^{12}$ was used to represent the effects of ions and core electrons. The all electron FLAPW method was adopted for the determination of the magnetic moment, magnetocrystalline anisotropy energy, $E_{\mathrm{MCA}}$, and magnetostrictive coefficients $\lambda_{100}$ and $\lambda_{111}$. The core electrons are treated fully relativistically,

\footnotetext{
${ }^{a)}$ Electronic mail: zhang@uta.edu.
}

while the spin-orbit coupling term was invoked second variationally for the valence states. ${ }^{13}$

We placed Fe and Co atoms in the bcc and hcp lattices in a 16-atom supercell and calculated total energies of all nonequivalent distribution configurations. The lattice sizes and atomic positions were optimized according to the energy minimization procedures. To quantitatively describe the composition dependence of structural stability of FeCo alloys, we define their formation energies as

$$
E_{b}\left(\mathrm{Co}_{m} \mathrm{Fe}_{n}\right)=E_{\text {tot }}\left(\mathrm{Co}_{m} \mathrm{Fe}_{n}\right)-m \mu_{\mathrm{Co}}-n \mu_{\mathrm{Fe}},
$$

where $E_{\text {tot }}\left(\mathrm{Co}_{m} \mathrm{Fe}_{n}\right)$ is the total energy of $\mathrm{Co}_{m} \mathrm{Fe}_{n}$ alloy in the ground state configuration with $m$ Co atoms and $n \mathrm{Fe}$ atoms per cell. $\mu_{\mathrm{Co}}$ and $\mu_{\mathrm{Fe}}$ represent chemical potentials or practically total energies of pure hcp Co and bcc Fe metals. Clearly, alloys with negative $E_{b}$ are stable while those with positive $E_{b}$ tend to segregate apart. In good accordance with previous studies, ${ }^{14,15}$ it is found that only the bec lattice is stable for $\mathrm{Fe}_{m} \mathrm{Co}_{n}$ alloys $(m>n)$, as listed in Table I, without first neighbor of minority atoms. Strikingly, $E_{b}$ for the hcp $\mathrm{Co}_{8} \mathrm{Fe}_{8}$ is as large as $+1.73 \mathrm{eV}$ (not shown in the table) per supercell, indicating that the Fe-Co alloy rather forms separated phases.

One important finding is that chemically noncubic geometries are preferred in a wide composition range. ${ }^{14}$ Note that $\mathrm{Fe}_{11} \mathrm{Co}_{5}, \mathrm{Fe}_{12} \mathrm{Co}_{4}$, and $\mathrm{Fe}_{13} \mathrm{Co}_{3}$ can be arranged with the cubic symmetry, as sketched in the first row of Fig. 1. Nevertheless, these structures appear to be the least stable one among all possible geometries as long as there is no Co first neighborhood. For $\mathrm{Fe}_{12} \mathrm{Co}_{4}$ (or equivalently $\mathrm{Fe}_{3} \mathrm{Co}$ ), for instance, the $L 6_{0}$ structure is more stable than the cubic $D_{3}$

TABLE I. Formation energies $\left(E_{b}\right)$ for FeCo alloys as defined in Eq. (1) and their corresponding spin magnetic moments $(M)$ per atom.

\begin{tabular}{lcc}
\hline \hline System & $E_{b}(\mathrm{eV} /$ cell $)$ & $M\left(\mu_{B}\right)$ \\
\hline $\mathrm{Fe}_{8} \mathrm{Co}_{8}$ & -0.51 & 2.21 \\
$\mathrm{Fe}_{9} \mathrm{Co}_{7}$ & -0.50 & 2.26 \\
$\mathrm{Fe}_{10} \mathrm{Co}_{6}$ & -0.55 & 2.28 \\
$\mathrm{Fe}_{11} \mathrm{Co}_{5}$ & -0.49 & 2.29 \\
$\mathrm{Fe}_{12} \mathrm{Co}_{4}$ & -0.36 & 2.30 \\
$\mathrm{Fe}_{13} \mathrm{Co}_{3}$ & -0.23 & 2.31 \\
$\mathrm{Fe}_{14} \mathrm{Co}_{2}$ & -0.107 & 2.28 \\
$\mathrm{Fe}_{15} \mathrm{Co}_{1}$ & 0.028 & 2.22 \\
\hline \hline
\end{tabular}



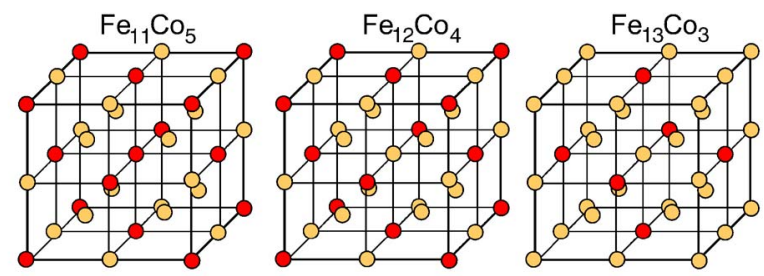

$\Delta \mathrm{E}=$
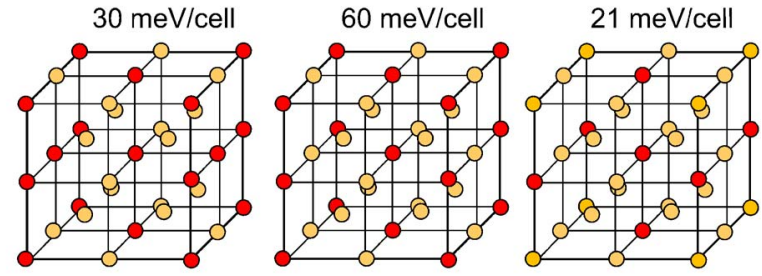

$\mathrm{E}_{\mathrm{MCA}}=$

$36 \mu \mathrm{eV} / \mathrm{cell}$

$-196 \mu \mathrm{eV} / \mathrm{cell}$

$8 \mu \mathrm{eV} / \mathrm{cell}$

FIG. 1. (Color online) Atomic configurations for the cubic (in the top row) and ground state (in the bottom row) structures of $\mathrm{Fe}_{11} \mathrm{Co}_{5}, \mathrm{Fe}_{12} \mathrm{Co}_{4}$ and $\mathrm{Fe}_{13} \mathrm{Co}_{3}$ alloys. The $\Delta E$ indicate the energy gains after the tetragonal distortion and the $E_{\mathrm{MCA}}$ are the magnetocrystalline anisotropy energies of the tetragonal structures.

structure by $60 \mathrm{meV} / \mathrm{cell}$ through VASP generalized gradient approximation (GGA) calculations or $70 \mathrm{meV} /$ cell through more precise FLAPW/GGA calculation. Since the phase transition can be achieved by swapping only one Co-Fe pair in the cell along either the (100) or (110) direction, it should be easy to occur if the sample is annealed.

The curves of density of states (DOS) for $\mathrm{Fe}_{12} \mathrm{Co}_{4}$ in both $L 6_{0}$ and $\mathrm{DO}_{3}$ structures are presented in Fig. 2. Obviously, the major difference occurs in $-1.8 \sim-1.0 \mathrm{eV}$ and $+1.5 \sim+2.0 \mathrm{eV}$, mostly on the Co sites. Corresponding to the large magnetic moments, the majority spin bands are fully occupied for both Co and Fe. The difference in DOS around the Fermi level is very small. However, the blip in the minority spin channel for the $\mathrm{DO}_{3}$ structure appears to be higher than that of the $L 6_{0}$ structure, which might be a cause for the slight instability of the former phase.

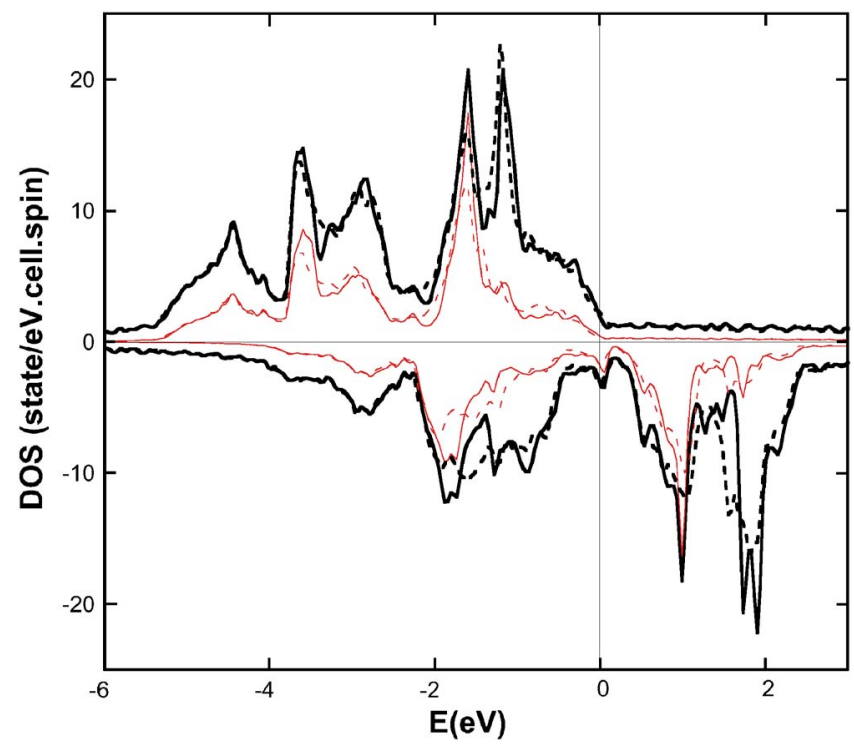

FIG. 2. (Color online) The calculated density of states of the $\mathrm{Fe}_{12} \mathrm{Co}_{4}$ in the $\mathrm{LG}_{0}$ (solid lines) and $\mathrm{DO}_{3}$ (dashed lines) structures. Contributions from the Co atoms are given in (thin lines). Positive and negative regions along the vertical axis are for the majority and minority spins, respectively. Zero en-

ergy is the position of the Fermi level.
Downloaded 11 Aug 2010 to 129.107.76.52. Redistribution subject to AIP license or copyright; see http://apl.aip.org/about/rights_and_permissions

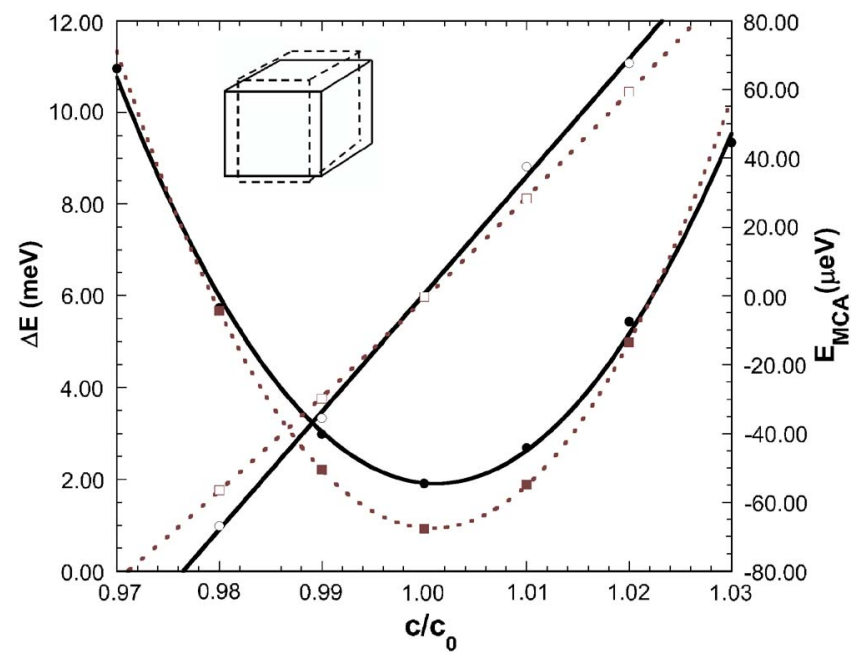

FIG. 3. (Color online) The calculated total energy $(\Delta E)$ and magnetocrystalline anisotropy energy $\left(E_{\mathrm{MCA}}\right)$ of $B 2$ FeCo alloy vs the lattice strain along the $\left(\begin{array}{lll}0 & 0 & 1\end{array}\right)$ direction. The solid-black curves are for $\Delta E$ with DFT optimized structure and the dashed-red curves are for the $3 \%$ expanded structure. The solid-black lines are for $E_{\mathrm{MCA}}$ with DFT optimized structure and the dashedred lines are for the $3 \%$ expanded structure.

As listed in Table I, the average magnetic moment varies in a small range for different compositions and appears to maximize at $m / n=3 / 1-4 / 1$. More explicitly for $\mathrm{Fe}_{12} \mathrm{Co}_{4}$ in the $L 6_{0}$ structure, the magnetic moment in Co is $1.80 \mu_{B}$, while those for two types of Fe atoms are $2.38 \mu_{B}$ (coplanar to $\mathrm{Co}$ ) and $2.63 \mu_{B}$ (in the pure Fe plane). Obviously, the reduction in magnetization by the presence of Co is excessively compensated by the strong enhancement of Fe magnetic moments. In addition, $\mathrm{Fe}$ and $\mathrm{Co}$ also contribute orbital magnetic moments in the size of $0.21-0.22 \mu_{B} /$ cell. The preference of noncubic structures has an important consequence: the production of uniaxial magnetocrystalline anisotropy energy (MAE). Through the torque approach, ${ }^{16}$ we found that the results of uniaxial MAE are large for $\mathrm{Fe}_{11} \mathrm{Co}_{5}$ and $\mathrm{Fe}_{12} \mathrm{Co}_{4}$ but remain small for $\mathrm{Fe}_{13} \mathrm{Co}_{3}$. Clearly, sizeable MAE can be attained in small grains or thin films that comprise pure noncubic phases.

The other source that may lead to sizeable magnetic anisotropy energy is the magnetoelastic interaction. Here, we applied strains along the (001) and (111) axes of the stoichiometric $B 2-\mathrm{Fe}_{0.5} \mathrm{Co}_{0.5}$ and adjusted the lattice constants in the perpendicular plane according to the constant-volume distortion mode. To take the effect of thermal expansion into account, we also investigated its properties under a 3\% lattice expansion. As shown in Fig. 3, the total energies and uniaxial magnetic anisotropy energies can be fitted by smooth functions of lattice strain, indicating the accuracy of our calculations. The calculated $\lambda_{001}$ and $\lambda_{111}$ reasonably agree with the experimental data ${ }^{17}$ (Table II), considering the fact that the atomic arrangement in the sample is more complex. Both $\lambda_{001}$ and $\lambda_{111}$ are large, especially under thermal expansions. This provides an effective venue for manipulation of MAE in FeCo. Finally, giant MAE (a few hundreds of $\mu \mathrm{eV}$ ) can be obtained if the lattice distortion is extended to the $10 \%-20 \%$ range. $^{18}$

In summary, the structural, electronic, and magnetic properties of $\mathrm{Fe}_{1-x} \mathrm{Co}_{x}$ alloys have been studied by firstprinciples methods. It is found that only the bcc lattice is thermodynamically stable for $x<0.5$. These alloys prefer chemically noncubic geometries in a wide composition range icense or copyright; see http://apl.aip.org/about/rights_and_permissions 
TABLE II. The calculated magnetostrictive coefficients in the $\left(\begin{array}{lll}0 & 0 & 1\end{array}\right)$ and ( $\left.\begin{array}{lll}1 & 1 & 1\end{array}\right)$ directions of $B 2 \mathrm{FeCo}$ alloy are compared with the experimental measurements. The one with structure expanded by $3 \%$ is also listed to show the tendency at thermal expansion.

\begin{tabular}{ccc}
\hline \hline & $\lambda_{001}\left(10^{-6}\right)$ & $\lambda_{111}\left(10^{-6}\right)$ \\
\hline $\begin{array}{c}\text { Discrete Fourier transform (DFT) } \\
\text { optimized structure }\end{array}$ & 88 & \\
3\% expanded structure & 122 & 30 \\
Experiment $^{\mathrm{a}}$ & 180 & 36 \\
\end{tabular}

${ }^{\mathrm{a}}$ Reference 17.

and, hence, possess large uniaxial MAE. From the sizeable magnetostrictive coefficients, MAE can be easily tuned in grains or films of FeCo alloys, where tetragonal or diagonal strains can reach $1 \%$.

This work was supported by the U.S. DoD/MURI under the Grant No. N00014-05-1-0497. R.W. thanks the support from the NSF (Grant No. DMR 0706503) and is also indebt to discussions with Dr. A. E. Clark, M. Won-Fungle, K. B. Hathaway, and Professor A. Flatau on the magnetostrictive properties. Calculations were performed on parallel computers at NERSC.

${ }^{1}$ F. Kneller and R. Hawig, IEEE Trans. Magn. 27, 3588 (1991).

${ }^{2}$ R. Skomski and J. M. D. Coey, IEEE Trans. Magn. 30, 607 (1996).
${ }^{3}$ Z. S. Shan, J. P. Liu, V. M. Chakka, H. Zeng, and J. S. Jiang, IEEE Trans. Magn. 38, 2907 (2002); Z. J. Guo, J. S. Jiang, J. E. Pearson, S. D. Bader, and J. P. Liu, Appl. Phys. Lett. 81, 2029 (2002).

${ }^{4}$ G. Asti, M. Solzi, M. Ghidini, and F. M. Neri, Phys. Rev. B 69, 174401 (2004).

${ }^{5}$ R. Skomski and J. M. D. Coey, Phys. Rev. B 48, 864 (1993); R. Skomski, J. Appl. Phys. 76, 7059 (1994).

${ }^{6}$ G. S. Chaubey, C. Barcena, N. Poudyal, C. Rong, J. Gao, S. Sun, and J. P. Liu, J. Am. Chem. Soc. 129, 7214 (2007).

${ }^{7}$ T. Burkert, L. Nordstrom, O. Eriksson, and O. Heinonen, Phys. Rev. Lett. 93, 027203 (2004).

${ }^{8}$ A. Winkelmann, M. Przybylski, F. Luo, Y. S. Shi, and J. Barthel, Phys. Rev. Lett. 96, 257205 (2006).

${ }^{9}$ G. Kresse and J. Hafner, Phys. Rev. B 49, 14251 (1994).

${ }^{10}$ E. Wimmer, H. Krakauer, M. Weinert, and A. J. Freeman, Phys. Rev. B 24, 864 (1981); M. Weinert, E. Wimmer, and A. J. Freeman, ibid. 26, 4571 (1982).

${ }^{11}$ J. P. Perdew, Phys. Rev. B 33, 8822 (1986); J. P. Perdew, K. Burke, and M. Ernzelhof, Phys. Rev. Lett. 77, 3865 (1996).

${ }^{12}$ P. E. Blöchl, Phys. Rev. B 50, 17953 (1994).

${ }^{13}$ R. Q. Wu and A. J. Freeman, J. Magn. Magn. Mater. 200, 498 (1999).

${ }^{14}$ R. Drautz, A. Díaz-Orbiz, M. Fähnle, and H. Dosch, Phys. Rev. Lett. 93, 067202 (2004); R. Drautz and A. Díaz-Orbiz, Phys. Rev. B 73, 224207 (2006).

${ }^{15}$ C. L. Fu and M. Krcmar, Phys. Rev. B 74, 174108 (2006).

${ }^{16}$ X. D. Wang, R. Q. Wu, and A. J. Freeman, Phys. Rev. B 54, 61 (1996).

${ }^{17}$ J. R. Cullen, A. E. Clark, and K. B. Hathaway, in Materials Science and Technology, edited by R. W. Cahn, P. Haasen, and E. J. Kramer (VCH, New York, 1994), Vol. 3B, p. 529.

${ }^{18}$ T. Burkert, L. Nordstrom, O. Eriksson, and O. Heinonen, Phys. Rev. Lett. 93, 027203 (2004). 